\title{
Comparison and Temporal Trends of Three Groups with Cryptococcosis: HIV-Infected, Solid Organ Transplant, and HIV-Negative/Non-Transplant
}

\author{
Emily W. Bratton ${ }^{1 *}$, Nada El Husseini ${ }^{2}$, Cody A. Chastain ${ }^{3}$, Michael S. Lee ${ }^{4}$, Charles Poole ${ }^{1}$, Til Stürmer ${ }^{1}$, \\ Jonathan J. Juliano ${ }^{4}$, David J. Weber ${ }^{1,4}$, John R. Perfect ${ }^{2}$
}

1 Department of Epidemiology, University of North Carolina at Chapel Hill, Chapel Hill, North Carolina, United States of America, 2 Department of Medicine, Duke University, Durham, North Carolina, United States of America, 3 Department of Medicine, Vanderbilt University Medical Center, Nashville, Tennessee, United States of America, 4 Department of Medicine, Brigham and Women's Hospital, Boston, Massachusetts, United States of America, $\mathbf{5}$ Department of Medicine, University of North Carolina at Chapel Hill, Chapel Hill, North Carolina, United States of America

\begin{abstract}
Background: The Infectious Disease Society of America (IDSA) 2010 Clinical Practice Guidelines for the management of cryptococcosis outlined three key populations at risk of disease: (1) HIV-infected, (2) transplant recipient, and (3) HIVnegative/non-transplant. However, direct comparisons of management, severity and outcomes of these groups have not been conducted.

Methodology/Principal Findings: Annual changes in frequency of cryptococcosis diagnoses, cryptococcosis-attributable mortality and mortality were captured. Differences examined between severe and non-severe disease within the context of the three groups included: demographics, symptoms, microbiology, clinical management and treatment. An average of nearly 15 patients per year presented at Duke University Medical Center (DUMC) with cryptococcosis. Out of 207 study patients, $86(42 \%)$ were HIV-positive, $42(20 \%)$ were transplant recipients, and 79 (38\%) were HIV-negative/non-transplant. HIV-infected individuals had profound CD4 lymphocytopenia and a majority had elevated intracranial pressure. Transplant recipients commonly (38\%) had renal dysfunction. Nearly one-quarter (24\%) had their immunosuppressive regimens stopped or changed. The HIV-negative/non-transplant population reported longer duration of symptoms than HIV-positive or transplant recipients and $28 \%(22 / 79)$ had liver insufficiency or underlying hematological malignancies. HIV-positive and HIV-negative/non-transplant patients accounted for $89 \%$ of severe disease cryptococcosis-attributable deaths and $86 \%$ of all-cause mortality.
\end{abstract}

Conclusions/Significance: In this single-center study, the frequency of cryptococcosis did not change in the last two decades, although the underlying case mix shifted (fewer HIV-positive cases, stable transplant cases, more cases with neither). Cryptococcosis had a relatively uniform and informed treatment strategy, but disease-attributable mortality was still common.

Citation: Bratton EW, El Husseini N, Chastain CA, Lee MS, Poole C, et al. (2012) Comparison and Temporal Trends of Three Groups with Cryptococcosis: HIVInfected, Solid Organ Transplant, and HIV-Negative/Non-Transplant. PLoS ONE 7(8): e43582. doi:10.1371/journal.pone.0043582

Editor: Michael Scheurer, Baylor College of Medicine, United States of America

Received March 27, 2012; Accepted July 23, 2012; Published August 24, 2012

Copyright: (c) 2012 Bratton et al. This is an open-access article distributed under the terms of the Creative Commons Attribution License, which permits unrestricted use, distribution, and reproduction in any medium, provided the original author and source are credited.

Funding: This work was supported by United States National Institutes of Health Public Health Service Grant [grant number Al73896]. The funders had no role in study design, data collection and analysis, decision to publish, or preparation of the manuscript.

Competing Interests: EWB, NEH, MSL, CAC, CP, JJJ and DJW have declared that they have no competing interests. The following authors have the following conflicts to declare: JRP has research grants, consulting and honorariums from Enzon, Pfizer, Merck, Astellas and Schering-Plough. TS does not accept personal compensation of any kind from any pharmaceutical company, though he receives salary support from the UNC Center of Excellence in Pharmacoepidemiology and Public Health and from unrestricted research grants from pharmaceutical companies to UNC. TS also receives research funding as Principal Investigator of the UNC-DEcIDE center from the Agency for Healthcare Research and Quality. However, this does not alter the authors' adherence to all the PLoS ONE policies on sharing data and materials.

* E-mail: emily_bratton@unc.edu

\section{Introduction}

Cryptococcus neoformans is an invasive mycoses that can cause meningoencephalitis, particularly among those who are immunocompromised, but in some cases it can infect immunocompetent individuals [1]. The 2010 IDSA Cryptococcal Guidelines defined three distinct risk groups for induction treatment of cryptococcosis [2]: (1) HIV-positive; (2) transplant recipients; and (3) a heterogeneous group with neither of these conditions (i.e., HIVnegative/non-transplant). A major component of this review was to describe outcomes of recent management of these three groups. During this study, four important factors were in play that justified our decision to use the broad 14-year study period in order to maximize cohort size. First, HAART became readily prescribed in 1996 with supportive evidence of the superiority of combination antiretroviral therapy over monotherapy in reducing AIDS morbidity and mortality [3]. Second, lipid products of amphotericin $\mathrm{B}$, for patients with renal impairment or unacceptable toxicity that prevent the use of conventional amphotericin $\mathrm{B}$, were in use 
since their initial FDA approval in November, 1995 [4]. Third, in 2000 the original IDSA Guidelines were published as a standard of treatment [5]. Fourth, there was an active Infectious Disease group at our institution with a particular interest in the pathogenesis and treatment of cryptococcosis.

The three risk groups defined by the IDSA and corresponding depths of information supporting treatment guidelines have been shaped through their individual patterns of emergence over time. In the last two decades, HIV-positive populations with cryptococcosis have been the most widely studied group $[6,7,8,9,10,11,12,13,14,15,16,17,18]$ and have received greater attention recently due to the recognition that cryptococcosis incidence in this group remains high, particularly in the AIDSinfected population in sub-Saharan Africa [19]. Starting in the 1960-1980's, use of immunosuppressive medications to treat severe diseases or for solid organ transplantation has increased the pool of patients susceptible to Cryptococcus. In the late 1990's, Cryptococcus gattii emerged in Vancouver Island, British Columbia, resulting in an outbreak of infections in both immunosuppressed and immunocompetent hosts [20,21]. The HIV-negative cryptococcosis patient group had been excluded from clinical review for several decades but has gained more attention recently [22,23,24,25,26,27]. Cryptococcal patients who are HIV-negative, particularly those who have few or no underlying risk factors (i.e., "apparently immunocompetent"), may experience more of a delay in time to presentation and diagnosis than HIV-positive or transplant recipient patients [26]. In particular, recent evidence has shown that HIV-negative, non-immunosuppressed cryptococcal meningitis patients suffered higher mortality rates than HIVpositive patients [25].

Due to its rare occurrence, prospective observational studies of this disease are logistically problematic. Interventional approaches have historically been based on expert opinion and retrospective cohort studies more than a decade old, with few representing HIVnegative populations and comparatively developed countries $[26,28,29,30]$. In this relatively large, retrospective single-center study, our goal was to provide an in-depth look at how cryptococcosis was managed clinically in the HIV-positive, transplant recipient and $\mathrm{HIV}$-negative/non-transplant patient groups in order to improve our understanding of this disease.

\section{Methods}

\section{Objectives}

The goals of this study were to describe trends in cryptococcosis symptoms, diagnosis, treatment and mortality through a 14-year study period (1996-2009) within the context of the three groups defined by the IDSA Guidelines.

\section{Participants}

We identified all consecutive adult patients ( $\geq 18$ years old) discharged from DUMC with International Classification of Diseases, 9th Revision (ICD-9) diagnosis codes of cryptococcosis (117.5), and cryptococcal meningitis (321.0) between January 1, 1996 and October 31, 2009 through electronic medical records. Eligible subjects had confirmed cryptococcal disease and a sufficient medical record (electronic and/or paper chart) available for review. A cryptococcosis case was confirmed by having $\geq 1$ of the following: positive cerebral-spinal fluid (CSF) cryptococcal antigen (CRAG) or fungal culture, direct histological examination of tissue or fluid with characteristic yeast forms of Cryptococcus, positive serum cryptococcal antigen test with a consistent disease process or positive culture from blood or pulmonary sites.

\section{Description of Investigations}

Demographics, presenting symptoms (including duration), and underlying conditions at the time of diagnosis were collected. Clinical differences examined included: presentation and duration of symptoms, microbiological evidence of cryptococcal disease, and initial antifungal treatment. Clinical isolates were not typed in this study. C. gattii is rare in the Southeastern U.S. with only one identified clinical case in an immunocompromised adult [31]. Serotype A (C. neoformans var. grubii) predominates the region of our study [32]. Follow-up visit information relevant to cryptococcosis (laboratory testing, clinic visits, and readmission) was also captured. Patients were followed from the date of diagnosis and/or admission until loss-to-follow-up, death, or until the end of the study period. In order to assess one-year mortality prevalence for all patients, we obtained data on survival and mortality up to one year after their date of cryptococcosis diagnosis from the Duke Data Support Repository (DSR), which uses the Social Security Administration death index, the Tumor Registry and The Duke Information System for Cardiovascular Care death data to report mortality status. Investigators recorded all information on a standardized abstraction form developed in collaboration with epidemiologists and clinicians.

We report on annual changes in frequency of cryptococcosis diagnoses, treatment, and outcomes including: overall mortality through one year, deaths attributable to cryptococcosis, and occurrence of immune reconstitution inflammatory syndrome (IRIS). Defined and determined by a panel of experts at DUMC, death was attributable if patients experienced conditions directly related to cryptococcal disease, such as: increased central nervous system (CNS) pressure, persistence or relapse of infection, while receiving initial induction treatment or due to organ failure during treatment for cryptococcosis. The criteria used to identify IRIS, adapted from Singh and Perfect (2007), included clinical or radiographic manifestations consistent with an inflammatory process, such as contrast enhancing lesions on imaging studies (CT/MRI), combined with symptoms that occurred during receipt of appropriate therapy and could not be explained by newly acquired infection, and at least one of the following: (1) negative results for cultures or stable/reduced biomarkers for the initial fungal pathogen during diagnostic work-up for the inflammatory process, (2) CSF pleocytosis $>5 \mathrm{WBC} / \mathrm{mm} 3$, (3) Increased ICP, (4) histopathology showing granulomatous lesions, or (5) unexplained hypercalcemia [33].

Central nervous system (CNS), pulmonary and 'other' cryptococcosis patients were collapsed into two categories based on specific indicators described in the 2010 IDSA Treatment Guidelines [2]: severe disease (evidence of CNS involvement, or cryptococcemia or dissemination with evidence of high fungal burden based on serum CRAG $\geq 1: 512$ ) or non-severe disease.

\section{Ethics}

This study was approved by both the Duke University Medical Institutional Review Board (IRB) and the University of North Carolina at Chapel Hill Biomedical IRB. Both named IRBs waived the need for informed consent for this study. This research met criteria for a waiver of informed consent according to Title 45, Code of Federal Regulations (CFR) Part 46.116(d).

\section{Statistical Methods}

All data was entered into Microsoft Office Access (2007) and analyses were performed using SAS v9.2 (SAS Institute, Cary, $\mathrm{NC}$ ). Variables were examined using descriptive statistics and stratified based on the three groups and/or severity of 
cryptococcosis as needed. Where appropriate, the Student's ttest was used to test the difference of two means and the Kruskal-Wallis test was used for the difference between medians for non-parametric continuous data. Chi-square $\left(X^{2}\right)$ tests were used to examine differences between categorical frequency distributions. The statistical significance level of alpha $(\alpha)$ equal to 0.05 was used for each two-tailed test performed, thus a "significant" result refers to a p-value $<0.05$.

\section{Results}

There were 223 study patients identified; 16 were excluded due to the following reasons: unable to locate chart $(\mathrm{n}=1)$, transferred out of care prior to diagnostic test results and did not receive treatment at DUMC $(n=4)$, patient $<18$ years $(n=1)$, and the patient did not have verified cryptococcal disease $(n=10)$. A total of 207 patients were used for analysis. The majority of cases were CNS disease $(61 \%)$, followed by pulmonary $(34 \%)$ and other sites (Table 1). Consistent across the three clinical groups, nearly twothirds of the cohort were male (65\%) and African Americans were more prevalent among HIV-positive patients than the other two groups (Table 1). HIV-positive patients were significantly younger than the other two groups.

Overall annual case frequencies of cryptococcosis did not significantly change over time (Figure 1). During the study period, the number of transplant patients per year averaged three (range, 0-5 cases/yr.). The frequency of HIV-infected cases averaged six annually (range, 2-12 cases/yr.). Among HIV-negative, nontransplant cases there was a slight increasing trend with time; the annual average number of cases was six (range, 3-9 cases/yr.). Although the total cases have remained relatively steady $(\sim 15 /$ $\mathrm{yr}$.), there appeared to be a shift to a decreasing proportion of HIV-positive patients with a concomitant increase in HIVnegative cases. HIV-positive patients accounted for half of all cases in the first seven years of this study then fell to less than onethird in the latter seven years.

Table 1. Patient characteristics at baseline.

Table 1. Patient characteristics at baseline.

\begin{tabular}{|c|c|c|c|c|c|c|c|c|c|c|}
\hline \multirow{3}{*}{ Category } & \multirow{3}{*}{ Subcategory } & & & \multicolumn{6}{|c|}{ Underlying Condition $^{a}$} & \multirow[b]{3}{*}{ p-value } \\
\hline & & \multicolumn{2}{|c|}{ Total } & \multicolumn{2}{|c|}{ HIV } & \multicolumn{2}{|c|}{ Transplant } & \multicolumn{2}{|c|}{ HIV-/Trans- ${ }^{b}$} & \\
\hline & & $\mathbf{N}$ & (\%) & $\mathbf{n}$ & (\%) & $\mathbf{n}$ & (\%) & $\mathbf{n}$ & $(\%)$ & \\
\hline \multicolumn{11}{|c|}{ Primary Diagnosis } \\
\hline & CNS & 126 & $(61)$ & 74 & $(86)$ & 18 & $(43)$ & 34 & $(43)$ & $>0.05$ \\
\hline & Pulmonary & 71 & (34) & 9 & $(10)$ & 24 & $(57)$ & 38 & $(48)$ & \\
\hline & Other & 10 & $(5)$ & 3 & (4) & 0 & $(-)$ & 7 & (9) & \\
\hline \multicolumn{11}{|c|}{ Disease severity } \\
\hline & Severe & 131 & $(63)$ & 74 & $(86)$ & 18 & $(43)$ & 39 & (49) & $>0.05$ \\
\hline & Non-severe & 76 & $(37)$ & 12 & (14) & 24 & $(57)$ & 40 & (51) & \\
\hline \multicolumn{11}{|c|}{ Demographics } \\
\hline & Male Gender & 135 & $(65)$ & 55 & (64) & 28 & $(67)$ & 52 & (66) & $>0.05$ \\
\hline & Black Race & 106 & (53) & 69 & $(80)$ & 13 & (31) & 24 & (30) & $>0.05$ \\
\hline & Age $(y r s)^{d}$ & 47 & (15) & 40 & (9) & 50 & (14) & 54 & (18) & $<0.05$ \\
\hline
\end{tabular}

Primary diagnosis, disease severity, basic patient characteristics and underlying condition of cryptococcosis patients at $\mathrm{DUMC}(\mathrm{N}=207)$.

${ }^{a}$ There were 86 patients in the HIV group, 42 in transplant and 79 in HIV-negative, non-transplant.

${ }^{\mathrm{b}}$ HIV-negative and non-transplant.

${ }^{c}$ Cochran Mantel-Haenszel Chi-square test for a general association between the three groups; Kruskal-Wallis test for difference between median age was used. ${ }^{d}$ Instead of $\mathrm{n}(\%)$, mean (STD) are shown for age.

doi:10.1371/journal.pone.0043582.t001

\section{Within-group Observations}

Within the HIV-positive patient group $(\mathrm{n}=86), 74(86 \%)$ had CNS disease, $9(11 \%)$ had pulmonary cryptococcosis and three patients had another form of cryptococcosis (Table 1). Twentyseven $(31 \%)$ patients were newly identified as positive for HIV infection during their hospital admission for cryptococcosis. CD4 counts were available for $62(72 \%)$ patients during hospitalization (median, 22 cells $/ \mu \mathrm{L}$; range: $1-300$ cells $/ \mu \mathrm{L}$ ). Forty-two percent of HIV-positive patients with severe $(n=31)$ and non-severe $(n=5)$ cryptococcosis reported current or previous exposure to HAART therapy, but management compliance was heterogeneous. Prevalence of reported HAART exposure did not differ significantly comparing earlier (1996-2002) and later cases (2003-2009). During admission 18 patients $(21 \%)$ continued their known Therapy, four patients $(5 \%)$ changed to another regimen, pix patients (7) continued their current therapy but changed held on admission. Seventeen patients $(20 \%)$ had no confirmed previous exposure to HAART; eight died with cryptococcal disease-related deaths. Thirty-five patients $(41 \%)$ were started newly on HAART therapy at the time of or after discharge. The median time-to-start of HAART therapy was 67 days after the start of induction therapy.

Among transplant recipients $(\mathrm{n}=42), 18(43 \%)$ had CNS disease (severe) and $24(57 \%)$ had pulmonary disease (non-severe; Table 1). The majority of transplants were renal $(n=17 ; 4$ included pancreas) followed by cardiac $(n=11)$ and pulmonary $(n=9)$. The median time from transplant to diagnosis of cryptococcosis $(\mathrm{n}=41)$ was 26 months (Inter-Quartile Range [IQR]: 10-56 months). Over one-third ( $\mathrm{n}=16$ ) of transplant recipients had renal insufficiency at the time of diagnosis (Table 2), but only two patients $(11 \%)$ with severe disease experienced a $>50 \%$ decrease in Glomerular Filtration Rate (GFR) during induction treatment. Current steroid exposure at the time of cryptococcosis was high (93\%), and 19 patients (24\%) had their immunosuppressive therapy stopped or changed at the time of their cryptococcosis diagnosis. Of the 39 patients taking steroids prior to cryptococcosis 


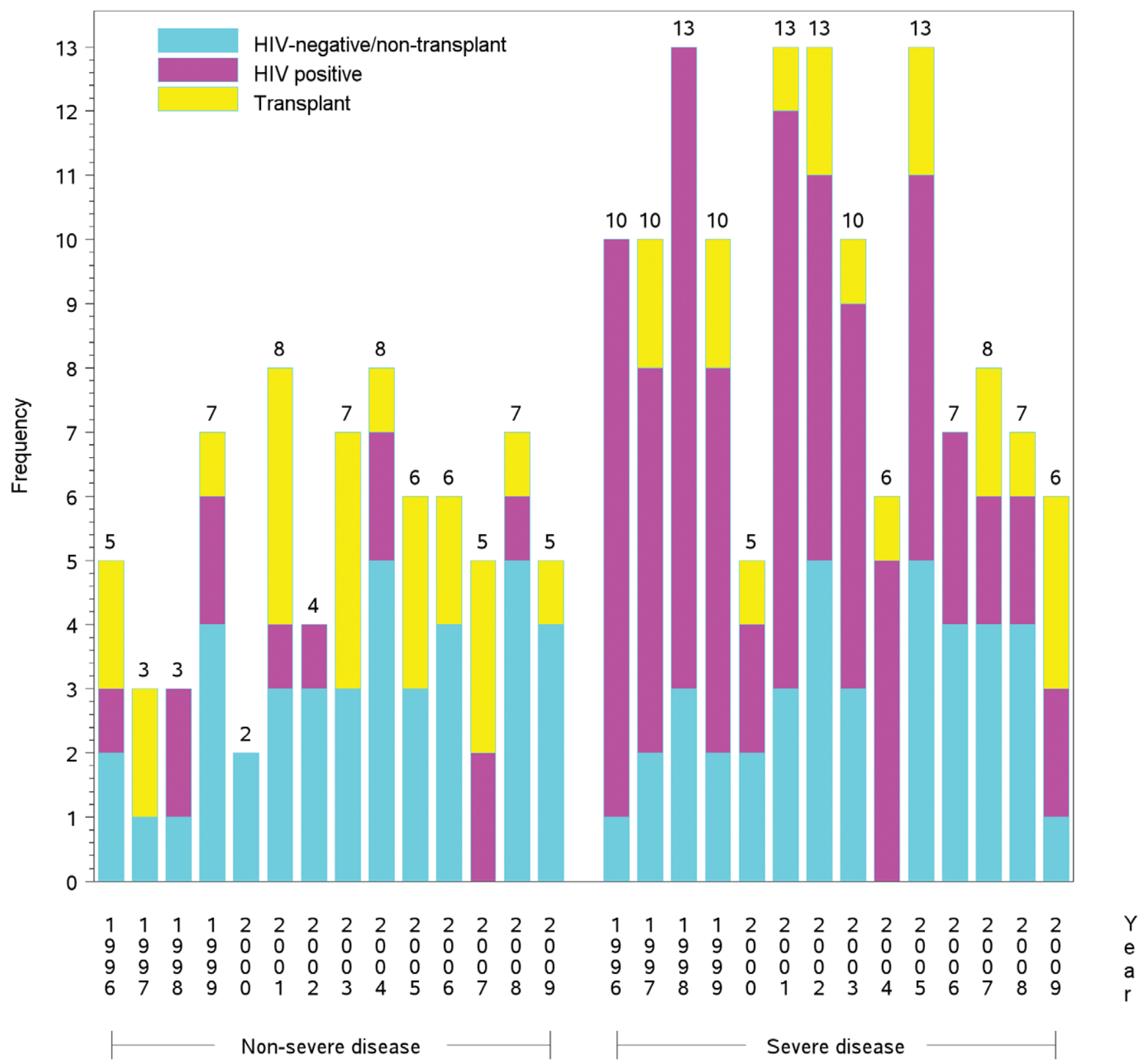

Figure 1. Annual cases. Annual frequency of severe and non-severe cryptococcosis cases according to underlying condition ( $\mathrm{N}=207)$. doi:10.1371/journal.pone.0043582.g001

diagnosis, 37 (95\%) had dose information. The median daily dose was $10 \mathrm{mg}$ of prednisone or prednisone equivalent (range: 4 $30 \mathrm{mg}$ /day). Most of these patients had been on extended immunosuppressive therapy. The median duration of immunosuppression $(\mathrm{n}=39)$ was 19 months (IQR: 8-56 months).

In the HIV-negative/non-transplant group $(\mathrm{n}=79), 34(43 \%)$ had CNS disease, $38(48 \%)$ had pulmonary cryptococcosis and 7 $(9 \%)$ had another form of cryptococcosis (Table 1). There were 37 $(47 \%)$ patients with no underlying malignancy or immunosuppressive therapy at the time of diagnosis. Ninety percent of all cancers in the cohort were among HIV-negative, non-transplant patients (Table 2). Of the 31 patients (39\%) taking steroids prior to cryptococcosis diagnosis, $24(77 \%)$ had dose information. The median daily dose was $20 \mathrm{mg}$ prednisone or prednisone equivalent (range: 5-267 mg/day). The median duration of any type of immunosuppressive therapy $(\mathrm{N}=21)$ was 7 months (IQR: $1-36$ months). Eight patients (21\%) with severe disease experienced a $>50 \%$ decrease in GFR during induction treatment.

\section{Clinical Symptoms}

There were 21 patients $(10 \%)$ who were asymptomatic at the time of diagnosis (Table 2). Of the 19 with asymptomatic pulmonary disease, 10 were transplant recipients and nine were HIV-negative/non-transplant patients.

Among the patients reporting symptoms $(n=186)$, the duration of symptoms was unknown for 30 patients; 10 (12\%) patients among those with HIV, seven $(22 \%)$ of transplant recipients and $13(18 \%)$ of the patients in the third group. Excluding these patients, the mean length of symptoms prior to presentation was not significantly different between severe and non-severe disease. The mean symptom duration was longer for the HIV-negative/ non-transplant patients than either of the other two groups 
Table 2. Presenting symptoms and risk factors of cryptococcosis disease $(\mathrm{N}=207)$.

\begin{tabular}{|c|c|c|c|c|c|c|c|c|c|c|c|c|c|c|c|}
\hline \multirow{3}{*}{ Presentation } & \multirow{3}{*}{ Characteristic } & \multicolumn{7}{|c|}{ Severe disease $(n=131)^{a}$} & \multicolumn{7}{|c|}{ Non-severe disease $(n=76)^{a}$} \\
\hline & & \multicolumn{2}{|c|}{ HIV } & \multicolumn{2}{|c|}{ Transplant } & \multicolumn{2}{|c|}{$\begin{array}{l}\text { HIV-I } \\
\text { Trans- }\end{array}$} & \multirow[t]{2}{*}{${ }^{*} p<0.05$} & \multicolumn{2}{|c|}{ HIV } & \multicolumn{2}{|c|}{ Transplant } & \multicolumn{2}{|c|}{$\begin{array}{l}\text { HIV-I } \\
\text { Trans- }\end{array}$} & \multirow[t]{2}{*}{${ }^{*} p<0.05$} \\
\hline & & $\mathbf{n}$ & $(\%)$ & $\mathbf{n}$ & (\%) & $\mathbf{n}$ & (\%) & & $\mathbf{n}$ & $(\%)$ & $\mathbf{n}$ & $(\%)$ & $\mathbf{n}$ & (\%) & \\
\hline \multicolumn{16}{|l|}{ Symptoms } \\
\hline & No symptoms & 1 & (1) & 0 & $(-)$ & 0 & $(-)$ & & 1 & (8) & 10 & (42) & 9 & (23) & \\
\hline & Altered mental status ${ }^{\mathrm{b}}$ & 22 & (30) & 7 & (39) & 17 & (44) & & 0 & $(-)$ & 1 & (4) & 0 & $(-)$ & \\
\hline & Headache & 54 & (73) & 9 & (50) & 17 & (44) & * & 2 & (17) & 3 & (13) & 2 & (5) & \\
\hline & Cough & 15 & (20) & 1 & (6) & 3 & (8) & & 7 & (58) & 3 & (13) & 11 & (28) & * \\
\hline & $\begin{array}{l}\text { Shortness of } \\
\text { breath }\end{array}$ & 10 & (14) & 1 & (6) & 5 & (13) & & 6 & (50) & 5 & (21) & 14 & (35) & \\
\hline & Night sweats & 5 & (7) & 2 & (11) & 1 & (3) & & 4 & (33) & 2 & (8) & 4 & (10) & \\
\hline & Fever & 40 & (54) & 7 & (39) & 12 & (31) & & 6 & (50) & 4 & (17) & 15 & (38) & \\
\hline & Nausea & 33 & (45) & 11 & (61) & 6 & (15) & * & 2 & (17) & 5 & (21) & 5 & (13) & \\
\hline & Vomiting & 28 & (38) & 8 & (44) & 5 & (13) & * & 3 & (25) & 3 & (13) & 3 & (8) & \\
\hline & Seizures & 8 & (11) & 0 & $(-)$ & 3 & (8) & & 0 & $(-)$ & 0 & $(-)$ & 1 & (3) & \\
\hline \multicolumn{16}{|c|}{ Condition/risk factor } \\
\hline & $\begin{array}{l}\text { Renal } \\
\text { insufficiency }\end{array}$ & 3 & (4) & 10 & (56) & 5 & (13) & * & 3 & (25) & 6 & (25) & 6 & (15) & \\
\hline & $\begin{array}{l}\text { Liver } \\
\text { insufficiency }\end{array}$ & 1 & (1) & 0 & $(-)$ & 6 & (15) & ${ }^{*}$ & 1 & (8) & 0 & $(-)$ & 2 & (5) & \\
\hline & $\begin{array}{l}\text { Hematologic } \\
\text { malignancy }\end{array}$ & 0 & $(-)$ & 1 & (6) & 7 & (18) & * & 0 & $(-)$ & 0 & $(-)$ & 7 & (18) & * \\
\hline & $\begin{array}{l}\text { Non-hematologic } \\
\text { Malignancy }\end{array}$ & 0 & $(-)$ & 0 & $(-)$ & 2 & (5) & & 1 & (8) & 0 & $(-)$ & 3 & (8) & \\
\hline \multicolumn{16}{|c|}{ Immunosuppressants } \\
\hline & Corticosteroid & 4 & (5) & 17 & (94) & 19 & (49) & * & 1 & (8) & 22 & (92) & 12 & (30) & * \\
\hline & $\begin{array}{l}\text { Calcineurin } \\
\text { inhibitor }\end{array}$ & 0 & $(-)$ & 15 & (83) & 0 & $(-)$ & * & 0 & $(-)$ & 21 & (88) & 2 & (5) & * \\
\hline & $\begin{array}{l}\text { Mycophenolate } \\
\text { mofetil }\end{array}$ & 0 & $(-)$ & 13 & (72) & 2 & (5) & * & 0 & $(-)$ & 10 & (25) & 2 & (5) & * \\
\hline & Azathioprine & 0 & $(-)$ & 2 & (11) & 1 & (3) & * & 0 & $(-)$ & 8 & (33) & 1 & (3) & * \\
\hline & Methotrexate & 0 & $(-)$ & 1 & (6) & 1 & (3) & & 0 & $(-)$ & 3 & (13) & 3 & (8) & \\
\hline & $\begin{array}{l}\text { Monoclonal } \\
\text { antibodies }\end{array}$ & 1 & (1) & 2 & (11) & 3 & (8) & & 0 & $(-)$ & 0 & $(-)$ & 2 & (5) & \\
\hline & Sirolimus & 0 & $(-)$ & 2 & (11) & 0 & $(-)$ & * & 0 & $(-)$ & 2 & (8) & 0 & $(-)$ & \\
\hline
\end{tabular}

Patients are stratified by disease severity at initial presentation and underlying condition.

a Severe disease: HIV group had 74 patients, transplant group had 18 patients and 39 patients were in the HIV-negative/non-transplant group; Non-severe disease: HIV group had 12 patients, transplant group had 24 patients and 40 patients were in the HIV-negative/non-transplant group.

${ }^{\mathrm{b}}$ Significantly associated with increased attributable-mortality.

${ }^{\text {cC}}$ Cochran Mantel-Haenszel Chi-square test for a general association between the three groups.

doi:10.1371/journal.pone.0043582.t002

(Table 3). When compared to this group, the difference in means was -25 days $(95 \%$ Confidence Limits [CL] - 50, 1) for the HIVpositive patients and -20 days $(95 \%$ CL $-48,8)$ for transplant recipients with severe disease. The differences in means were even greater when comparing the groups with non-severe disease (Table 3).

Patients with severe disease frequently experienced headaches, altered mental status, fevers, nausea and vomiting across all three groups at initial presentation (Table 2). The prevalence of nausea and vomiting was significant between groups with them being more common in HIV and transplant recipients. Furthermore, headache was significantly more prevalent among HIV-positive patients $(73 \%)$ and similar between the other two groups. Symptoms among non-severe cryptococcosis patients were similar between transplant recipients and HIV-negative/non-transplant groups. However, the prevalence of corticosteroid exposure was significantly higher in transplant recipients (Table 2).

\section{Patient Diagnostics}

Among patients with severe disease, patients had similar lumbar puncture (LP) results among all three groups (Table 4). At least one opening pressure $(\mathrm{OP})$ measurement was available for $79(63 \%)$ patients. Peak OP distributions were very similar between all three groups with a mean of $33 \mathrm{~cm} \mathrm{H}_{2} \mathrm{O}\left(\mathrm{SD} \pm 16 \mathrm{~cm} \mathrm{H}_{2} \mathrm{O}\right)$. The proportion of $\mathrm{HIV}$-positive patients (48\%) with elevated CSF host cells $\left(\geq 20\right.$ cells $/ \mathrm{mm}^{3}$ ) was significantly less than the other groups, which had frequencies of $78 \%$ and $61 \%$ (Table 4$)$. The difference in frequencies across all three patient groups having an elevated 
Table 3. Differences in mean duration of symptoms (days) reported prior to presentation among those reporting any symptom(s) of cryptococcosis $(\mathrm{N}=156)$.

\begin{tabular}{|c|c|c|c|c|c|c|c|c|}
\hline \multirow{2}{*}{ Underlying condition } & \multicolumn{4}{|c|}{ Severe disease } & \multicolumn{4}{|c|}{ Non-severe disease } \\
\hline & $\mathbf{n}$ & Mean & Difference & $95 \% \mathrm{CL}^{\mathrm{a}}$ & $\mathbf{n}$ & Mean & Difference & $95 \% \mathrm{CL}^{\mathrm{a}}$ \\
\hline HIV-negative, non-transplant & 36 & 44 & 0 (ref.) & 20,68 & 21 & 66 & 0 (ref.) & 29,103 \\
\hline HIV positive & 65 & 19 & -25 & $-50,1$ & 10 & 26 & -40 & $-80,0$ \\
\hline Transplant & 14 & 24 & -20 & $-48,8$ & 11 & 20 & -46 & $-84,-8$ \\
\hline Total & 114 & 28 & & 19,37 & 42 & 44 & & 24,64 \\
\hline
\end{tabular}

anadjusted $95 \%$ Confidence Limits (CL) of the difference in means. The $95 \% \mathrm{CL}$ for the referent group and the total are surrounding their corresponding mean (days). doi:10.1371/journal.pone.0043582.t003

CSF CRAG titer $(\geq 1: 1024)$ and positive India ink neared significance. With regard to the frequency of these two CSF diagnostic measures, further comparison of non-HIV/non-transplant with the other two groups combined (differences in frequencies between HIV-positive and transplant patients were not significant) indeed reached the level of significance. CSF glucose and protein levels were similar in all groups $(\sim 40 \%$ of all patients had hypoglycorrhachia). There was no significant difference between groups in regards to cryptococcemia (Table 4).
Among non-severe disease patients, histological evidence of Cryptococcus was identified in over $60 \%$ of the two HIV-negative groups (Table 4). Positive pulmonary cultures were identified by at least one positive culture, biopsy or broncho-alveolar lavage in $>50 \%$ of HIV-negative patients, whereas only $17 \%(\mathrm{n}=2)$ of HIV-positive patients had documentation of pulmonary disease. Of the $40 \mathrm{HIV}$-negative/non-transplant patients, 22 (55\%) received an LP to rule out CNS disease, which was not significant compared to the $18(75 \%)$ transplant recipients but was significant compared to the $11(92 \%)$ HIV-positive patients who received an

Table 4. Diagnostic findings of cryptococcosis disease $(\mathrm{N}=207)$ stratified by disease severity at initial presentation and underlying condition.

\begin{tabular}{|c|c|c|c|c|c|c|c|c|c|c|c|c|c|c|c|}
\hline \multirow{3}{*}{ Diagnostic } & \multirow{3}{*}{ Result description } & \multicolumn{7}{|c|}{ Severe disease $(n=131)^{a}$} & \multicolumn{7}{|c|}{ Non-severe disease $(n=76)^{a}$} \\
\hline & & \multicolumn{2}{|c|}{ HIV } & \multicolumn{2}{|c|}{ Transplant } & \multicolumn{2}{|c|}{ HIV-/Trans- } & \multirow{2}{*}{${ }^{*} p<0.05$} & \multicolumn{2}{|c|}{ HIV } & \multicolumn{2}{|c|}{ Transplant } & \multicolumn{2}{|c|}{ HIV-/Trans- } & \multirow[t]{2}{*}{${ }^{*} \mathbf{p}<0.05$} \\
\hline & & $\mathbf{n}$ & (\%) & $\mathbf{n}$ & (\%) & $\mathbf{n}$ & (\%) & & $\mathbf{n}$ & (\%) & $\mathbf{n}$ & (\%) & $\mathbf{n}$ & (\%) & \\
\hline \multicolumn{16}{|c|}{ Positive culture } \\
\hline & $C N S$, first $L P^{b}$ & 61 & (82) & 18 & (100) & 22 & (56) & & 0 & - & 0 & - & 0 & - & \\
\hline & Blood $^{c}$ & 35 & (47) & 8 & (44) & 11 & (28) & & 6 & (50) & 2 & (8) & 5 & (13) & * \\
\hline & Pulmonary & 6 & (8) & 2 & (11) & 3 & (8) & & 2 & (17) & 13 & (54) & 21 & (53) & \\
\hline \multicolumn{16}{|l|}{ Initial LP } \\
\hline & CSF CRAG titer $\geq 1: 1024$ & 35 & (48) & 6 & (33) & 8 & (24) & & - & - & - & - & - & - & \\
\hline & $\begin{array}{l}\text { CSF:serum glucose ratio } \\
<0.6\end{array}$ & 66 & (89) & 16 & (89) & 28 & (85) & & - & - & - & - & - & - & \\
\hline & CSF glucose $\leq 40 \mathrm{mg} / \mathrm{dL}$ & 43 & (58) & 11 & (61) & 20 & (61) & & - & - & - & - & - & - & \\
\hline & CSF protein $\geq 45 \mathrm{mg} / \mathrm{dL}$ & 66 & (89) & 16 & (89) & 32 & (97) & & - & - & - & - & - & - & \\
\hline & $\begin{array}{l}\text { Nucleated cells }>20 \text { cells/ } \\
\mathrm{mm}^{3, \mathrm{~d}}\end{array}$ & 30 & (48) & 14 & (78) & 20 & (71) & * & & & & & & & \\
\hline & $\mathrm{OP} \geq 20 \mathrm{~cm} \mathrm{H}_{2} \mathrm{O}^{\mathrm{e}}$ & 34 & (76) & 3 & (38) & 17 & (52) & & - & - & - & - & - & - & \\
\hline & Positive India Ink & 41 & (55) & 9 & (50) & 11 & (33) & & - & - & - & - & - & - & \\
\hline \multicolumn{16}{|l|}{ Other } \\
\hline & Serum CRAG titer $\geq 1: 1024$ & 47 & (64) & 11 & (61) & 17 & (44) & & 5 & (42) & 3 & (13) & 4 & (10) & * \\
\hline & Other histological evidence & 9 & (12) & 3 & (17) & 6 & (18) & & 0 & - & 15 & (63) & 27 & (68) & * \\
\hline
\end{tabular}

a Severe disease: HIV group had 74 patients, transplant group had 18 patients and 39 patients were in the HIV-negative/non-transplant group; Non-severe disease: HIV group had 12 patients, transplant group had 24 patients and 40 patients were in the HIV-negative/non-transplant group.

${ }^{\mathrm{b}}$ Lumbar puncture; 176 total patients had an LP. Six HIV-negative, non-transplant patients with severe disease did not receive an LP and the remaining 25 patients without an LP were non-severe cases (18 HIV-negative/non-transplant, six transplant recipients, and one HIV-positive patient). Percentages relevant to the LP procedure reflect missing observations.

'Significantly associated with attributable-mortality.

dEleven (15\%) HIV-infected patients had missing documentation of CSF host (nucleated) cells (overall N=63); excluding the six with no initial LP, five HIV-negative, nontransplant were missing host cell counts (overall $\mathrm{N}=28$ ).

eOpening pressure; maximum LP OP was used for this variable, as initial LP OP was infrequently captured. Denominators for each group with severe disease were 74,8 , and 33 , respectively.

doi:10.1371/journal.pone.0043582.t004 
LP when non-severe disease was identified.

\section{Initial Treatment}

During the study period, 132 patients (64\%) received amphotericin B (either formulation) for initial induction therapy. Utilization of amphotericin B deoxycholate (AmpBd) decreased over time, indicating that lipid formulation amphotericin B (LFAmpB) was used more frequently as initial therapy in recent years (Figure 2). Despite this observed trend, AmpBd was used as initial induction therapy for $80 \%$ of patients across the entire study period.

Induction antifungal regimens are summarized in Table 5 . Eighty percent of patients with non-severe disease were given fluconazole for initial treatment; this was not significantly different across the three risk groups. In the severe disease group, the frequency of polyene use as initial therapy was high for all three patient groups $(89 \%$ of HIV-positive, $100 \%$ of transplant, and $87 \%$ of HIV-negative/non-transplant), as was the use of flucytosine in combination with the polyene $(78 \%$ of $\mathrm{HIV}$-positive, $83 \%$ of transplant, and $72 \%$ of HIV-negative/non-transplant).

\section{Mortality and IRIS}

Mortality attributable to cryptococcosis was $15 \%(\mathrm{n}=31)$ and there was a total of 52 deaths $(25 \%)$ through one year of follow-up (Table 5). HIV-positive and HIV-negative/non-transplant patients accounted for $89 \%$ of severe disease cryptococcosis-attributable deaths and these two groups accounted for $86 \%$ of all-cause mortality. IRIS was identified in seven $(3 \%)$ cases and most of these cases had severe cryptococcosis. Four out of the seven patients were HIV-positive but IRIS was observed in the other two groups.

The HIV-negative/non-transplant group experienced both greater mortality attributable to cryptococcosis and overall mortality, since they accounted for nearly half of all cryptococ- cosis-attributable deaths (15/31) and more than half of all-cause mortality (29/52). Within this group, patients who died were older at diagnosis (mean, 63 years) than those who did not (mean, 51 years). However, the average age at diagnosis was not significantly different between survivors and those who died within either the HIV-positive or transplant groups.

\section{Discussion}

The 2010 IDSA Guidelines divided cryptococcal disease into three risk groups because of their different management issues in an attempt to better describe the issues around treatment and outcome [2]. The results from our study found notable trends and important clinical differences between and within these groups and uniquely describes the realities in the management of this disease at one institution.

In the early parts of the 14-year study period, the highest number of cases occurred in the HIV-infected population, which appeared to experience fewer of cryptococcal infections in recent years, coinciding with the widespread use of HAART. However, $42 \%$ of HIV-positive patients in this cohort had been exposed to HAART, emphasizing that despite therapies to control HIV infection, cryptococcosis will continue to be an opportunistic infection in HIV-infected persons. The HIV-negative/non-transplant patients appeared to offset the reduction of cryptococcosis seen in HIV-infected patients in more recent years. As we continue to aggressively treat serious underlying diseases with immunosuppressants and the denominator of persons-at-risk enlarges, this group will likely increase since there is no strategy for prophylaxis. There was a consistent number of cases of cryptococcosis in the transplant recipients over time despite the widespread use of the potentially anti-cryptococcal agents, the calcineurin inhibitors $[34,35,36,37,38]$. The steady annual prevalence of cryptococcosis in this group supports the continued routine use of immunosup-

\section{Percent utilization of each amphotericin B formulation for initial cryptococcosis therapy by year of diagnosis $(n=132)$}

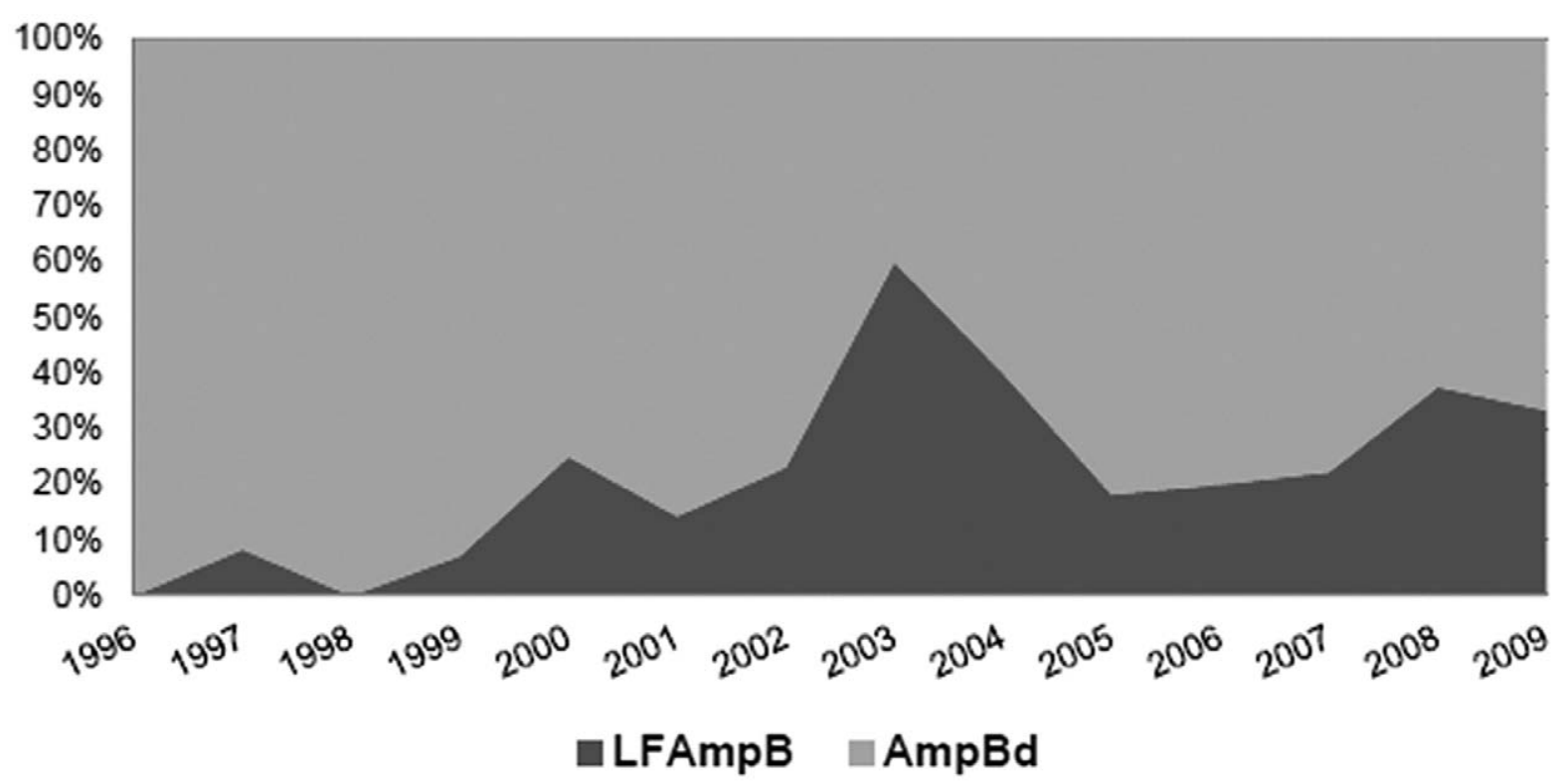

Figure 2. Use of amphotericin B. Amphotericin $B$ formulation trends over time. Annual percentage of patients receiving lipid formulation amphotericin B (LF AmpB) or deoxycholate amphotericin B (AmpBd) for initial therapy $(N=132)$. doi:10.1371/journal.pone.0043582.g002 
Table 5. Patient treatment and outcomes.

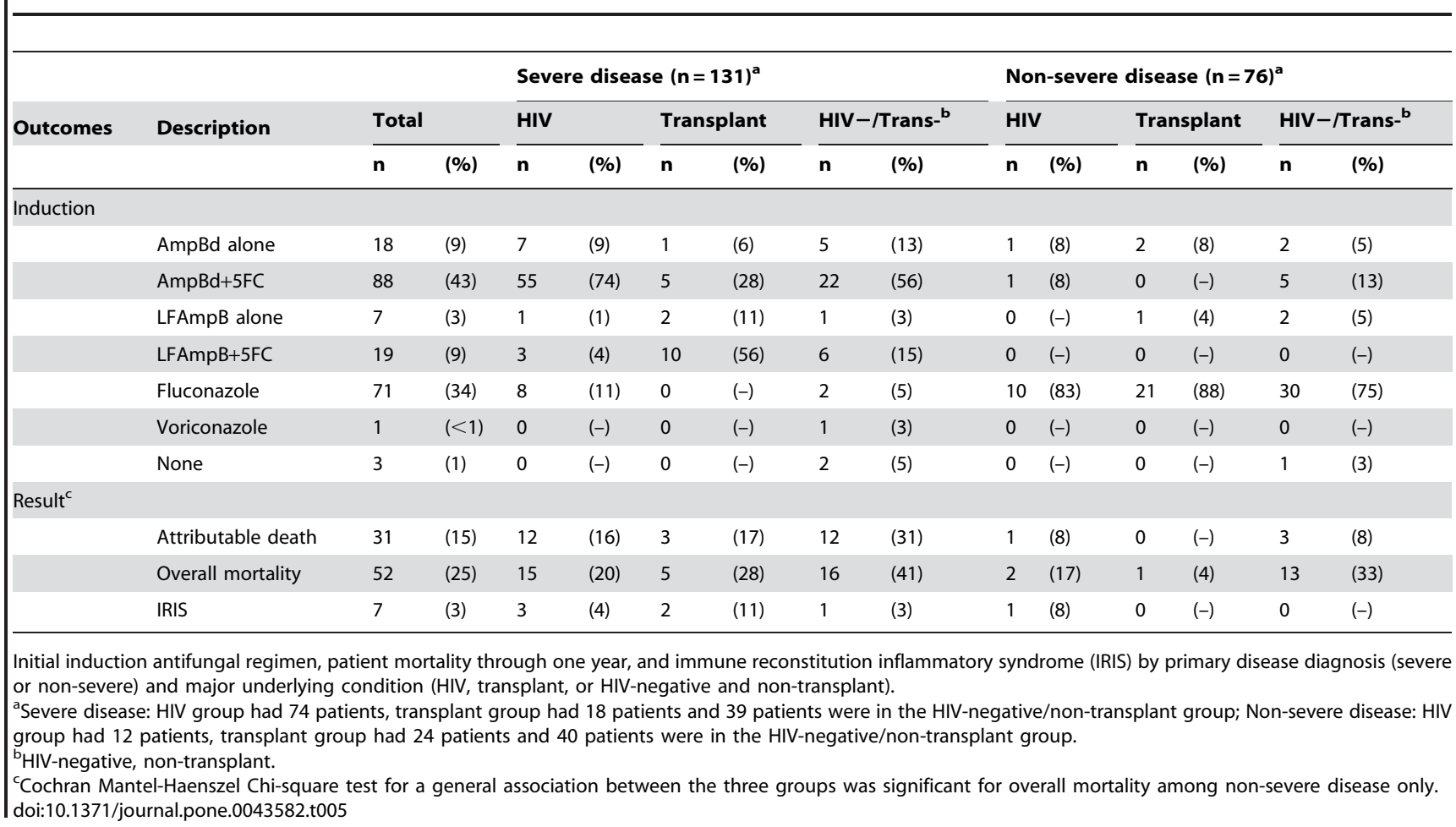

pressants and thus a persistent need for careful diagnostic surveillance for detection of early cryptococcosis [39].

The differences within the groups were several. For HIVinfected individuals, there was a variety of antiretroviral strategies employed during anti-cryptococcal therapy that reflects the lack of precise guidelines on when to initiate HAART [40]. Similar to previous studies, most of these patients had profound CD4 lymphocytopenia and a majority of these patients $(76 \%)$ had elevated intracranial pressure $[16,17,41,42]$. The important underlying issues surrounding transplant recipients were immunosuppressive drugs and frequent renal dysfunction. All had some form of immunosuppression but only one-quarter had their immunosuppressive regimens stopped or changed and the prevalence of IRIS was low. Also, one-third of patients started treatment with evidence of renal dysfunction, emphasizing that lipid products of amphotericin B may be essential therapeutic choices in this group and that monitoring flucytosine levels and/or complete blood counts may be necessary to prevent treatment toxicity during worsening of renal dysfunction caused by polyene treatment [43]. Moreover, the average time from transplant to cryptococcal infection was within range of the 17-28 months reported in previous studies $[37,43,44,45,46]$. There were two important findings in the HIV-negative/non-transplant group. First, the duration of symptoms in this group with severe disease averaged 44 days prior to diagnosis and although not reaching significance from the other groups (it was significant among those with non-severe disease), this notable delay deserves greater attention and has been observed in a previous case series [23]. This delay may have contributed to the observed poorer outcome of the group. Another study also found a lack of significance between HIV-infected, immunocompromised, and immunocompetent groups, where the symptom duration averaged approximately 15 days [25]. It is possible that the other two groups have specialists aware of the risk of cryptococcosis, while in this group diagnosis is delayed because cryptococcosis is not considered.
Second, 33\% had liver insufficiency or hematological malignancies. These are important findings as this subgroup had the highest mortality and both factors have been shown to be predictors of mortality in HIV-negative cryptococcosis patients [24,47]. Previous results emphasize that disseminated cryptococcosis among HIV-negative patients experienced the worst prognosis secondary to the stage of underlying disease and the immunosuppressive medication used [26,34]. It has been clearly shown in the HIVpositive population with cryptococcal disease that stage of HIV is strongly associated with poor outcome [48]. The underlying disease and its stage are major factors in cryptococcosis outcome.

Since delay in diagnosis may be a prognostic factor, we investigated whether there were additional differences in symptoms and laboratory findings between the three groups. There were few differences between HIV-infected patients and transplant recipients who generally had similar symptoms and CSF laboratory parameters. However, headaches (known to be a prognostic factor) [29], were similar between both HIV-negative groups and significantly more prevalent in the HIV-positive group. While all three groups had a high prevalence of poor prognostic signs such as altered mental status $(\sim 1 / 3)$, the presence of nausea and vomiting were less common in the HIV-negative/nontransplant group with severe disease. These findings differ from another study that reported significantly more mental status changes in non-immunosuppressed patients compared to HIVpositive patients [25]. Additionally, our study supports previous evidence that suggests non-immunosuppressed patients have less fungemia [24]. The HIV-negative/non-transplant group also appeared to present with a smaller burden of yeasts by India ink and CRAG test results than the other two groups. Although appreciation for burden of organisms and outcome could not be precisely understood in this retrospective review, prospective studies of cryptococcal meningitis may benefit from quantification of viable yeasts in the CSF (Colony-forming unit $[\mathrm{CFU}] / \mathrm{mL}$ measurements) and understanding its rate of change during 
therapy in relationship to treatment strategy and outcome $[12,49,50]$.

Importantly, compared to HIV-positive and transplant recipients, the attributable mortality in the HIV-negative/non-transplant population with severe disease was nearly two-times higher despite the fact that the majority of patients received induction therapy with a polyene and flucytosine. A recent multi-center study of 86 cryptococcal meningitis patients also found the highest mortality in the non-immunosuppressed group (46\%) compared to immunosuppressed (19\%) and HIV-positive (15\%) cryptococcal meningitis patients [25], and previous studies have reported 30 $44 \%$ overall mortality in the HIV-negative population $[22,23,24,26]$. In one of these studies this rate was compared to a $22 \%$ mortality among HIV-positive patients [24]. However, a couple of these studies included some C. gatii cases so that species' factors may have influenced outcome [22,24]. Clearly more studies to inform the management of the HIV-negative/nontransplant group and to understand how host immunity and yeast strain may play a role in poorer prognosis are needed so as to reduce this elevated mortality.

One of the major complications of cryptococcosis management has been the identification and management of IRIS. We identified the occurrence of IRIS in all three groups but the prevalence $(3 \%)$ was relatively low compared to other studies which primarily included AIDS patients (range, 8-19\%) $[51,52,53,54]$. This frequency could be influenced by our definition of IRIS, the patient mix, and/or clinical management. In general, our HAART management was delayed $(>60$ days after start of induction therapy) and only approximately a quarter of the transplant recipients had their immunosuppressants adjusted. This lack of immune manipulation during early induction therapy may influence our lower rate of IRIS. However, it is identified in a measureable amount of all patient groups and needs to be appreciated by clinicians.

Lipid formulations of amphotericin B outside of HIV-infected patients have limited critical appraisal of proper dosing and efficacy [55,56]. However, as Figure 2 demonstrated, there is a general increase in the use of lipid formulations for induction therapy at our institution. We expect this was in relationship to the approximately third of patients who develop nephrotoxicity during management. Therefore, further investigation into lipid products of amphotericin B is still warranted to ensure their optimal use.

\section{Limitations}

This review was limited to a single tertiary care center and teaching hospital. Our medical center averaged nearly 15 cases of cryptococcosis per year and this likely reflects both an endemic exposure to this yeast in the environment within the Southeastern USA and an enriched population of immunosuppressed individuals due to our hospital's care patterns. The actual number of

\section{References}

1. Casadevall A, Perfect JR (1998) Cryptococcus neoformans. Washington, D.C.: ASM Press. viii, 541 p.

2. Perfect JR, Dismukes WE, Dromer F, Goldman DL, Graybill JR, et al. (2010) Clinical practice guidelines for the management of cryptococcal disease: 2010 update by the Infectious Diseases Society of America. Clin Infect Dis 50: 291322 .

3. Palella Jr FJ, Delaney KM, Moorman AC, Loveless MO, Fuhrer J, et al. (1998) Declining morbidity and mortality among patients with advanced human immunodeficiency virus infection. HIV Outpatient Study Investigators. N Engl J Med 338: 853-860.

4. Rust DM, Jameson G (1998) The novel lipid delivery system of amphotericin B: drug profile and relevance to clinical practice. Oncol Nurs Forum 25: 35-48.

5. Saag MS, Graybill RJ, Larsen RA, Pappas PG, Perfect JR, et al. (2000) Practice guidelines for the management of cryptococcal disease. Infectious Diseases Society of America. Clin Infect Dis 30: 710-718. cases seen in a particular medical center certainly varies within the U.S. Furthermore, using hospital records favors cases with severe disease and could result in selection bias against asymptomatic disease. Determining the total population-at-risk was not estimable in this study and the underlying source population and referral patterns could shift over time. Retrospective chart review has the potential for incomplete or incorrect information capture due to loss of paper documentation or lack of entry in electronic medical record. We used both sources to ensure data was as complete as possible and discrepancies were minimized. Erroneous self-report of symptoms or symptom duration was a possibility, but this is a limitation of many observational clinical studies. Despite our careful abstraction process, missing or incomplete data could lead to bias in categorization of symptoms or derived outcome definitions, such as IRIS. Being a rare disease, limited numbers of cases prevented robust statistical analyses, although this study is one of the largest to date. Importantly, much of the clinical attention over the last two decades has centered around two groups (HIV-infected and transplant recipients). There has been less focus on treatment of HIV-negative/non-transplant patients and yet this group suffered the highest mortality.

\section{Conclusions}

In summary, dividing patients with cryptococcosis into three risk groups showed both differences and similarities within the groups. In a single medical center the overall frequency of cryptococcosis has not changed though the composition of the three groups has changed in the last two decades. Despite three major classes of drugs to treat severe disease and a relatively uniform and informed treatment strategy framed by the IDSA Guidelines, attributable mortality was common. Prospective multicenter studies and comparison of strategies in advanced medical centers are still needed to determine the extent high mortality revolved around underlying disease, high burden of organisms and delayed diagnosis.

\section{Acknowledgments}

We would like to extend our gratitude to Elizabeth S. Dodds Ashley, PharmD, for assistance with initial study preparations such as abstraction tool revisions and database creation, as well as Mariza Daras, MD, and Lipi Roy, MD, MHS, for assisting with patient chart collection.

\section{Author Contributions}

Conceived and designed the experiments: EWB NEH CAC MSL JRP. Performed the experiments: EWB NEH CAC MSL. Analyzed the data: EWB. Wrote the paper: EWB. Interpretation of data: EWB CP TS JIJ DJW JRP. Selection of analytic tools: EWB CP TS JRP. Critical revision and editing of manuscript: CP TS JJJ DJW JRP.

6. Nussbaum JC, Jackson A, Namarika D, Phulusa J, Kenala J, et al. (2010) Combination flucytosine and high-dose fluconazole compared with fluconazole monotherapy for the treatment of cryptococcal meningitis: a randomized trial in Malawi. Clin Infect Dis 50: 338-344.

7. Jarvis JN, Dromer F, Harrison TS, Lortholary O (2008) Managing cryptococcosis in the immunocompromised host. Curr Opin Infect Dis 21: 596-603.

8. Pappas PG, Chetchotisakd P, Larsen RA, Manosuthi W, Morris MI, et al. (2009) A phase II randomized trial of amphotericin B alone or combined with fluconazole in the treatment of HIV-associated cryptococcal meningitis. Clin Infect Dis 48: 1775-1783.

9. Bicanic T, Wood R, Meintjes G, Rebe K, Brouwer A, et al. (2008) High-dose amphotericin $\mathrm{B}$ with flucytosine for the treatment of cryptococcal meningitis in HIV-infected patients: a randomized trial. Clin Infect Dis 47: 123-130. 
10. Longley N, Muzoora C, Taseera K, Mwesigye J, Rwebembera J, et al. (2008) Dose response effect of high-dose fluconazole for HIV-associated cryptococcal meningitis in southwestern Uganda. Clin Infect Dis 47: 1556-1561.

11. Milefchik E, Leal MA, Haubrich R, Bozzette SA, Tilles JG, et al. (2008) Fluconazole alone or combined with flucytosine for the treatment of AIDSassociated cryptococcal meningitis. Med Mycol 46: 393-395.

12. Brouwer AE, Rajanuwong A, Chierakul W, Griffin GE, Larsen RA, et al. (2004) Combination antifungal therapies for HIV-associated cryptococcal meningitis: a randomised trial. Lancet 363: 1764-1767.

13. Dromer F, Mathoulin-Pelissier S, Fontanet A, Ronin O, Dupont B, et al. (2004) Epidemiology of HIV-associated cryptococcosis in France (1985-2001): comparison of the pre- and post-HAART eras. AIDS 18: 555-562.

14. French N, Gray K, Watera C, Nakiyingi J, Lugada E, et al. (2002) Cryptococcal infection in a cohort of HIV-1-infected Ugandan adults. AIDS 16: 1031-1038.

15. Hajjeh RA, Conn LA, Stephens DS, Baughman W, Hamill R, et al. (1999) Cryptococcosis: population-based multistate active surveillance and risk factors in human immunodeficiency virus-infected persons. Cryptococcal Active Surveillance Group. J Infect Dis 179: 449-454.

16. Kaplan JE, Hanson D, Dworkin MS, Frederick T, Bertolli J, et al. (2000) Epidemiology of human immunodeficiency virus-associated opportunistic infections in the United States in the era of highly active antiretroviral therapy. Clin Infect Dis 30 Suppl 1: S5-14.

17. Mirza SA, Phelan M, Rimland D, Graviss E, Hamill R, et al. (2003) The changing epidemiology of cryptococcosis: an update from population-based active surveillance in 2 large metropolitan areas, 1992-2000. Clin Infect Dis 36: 789-794.

18. van der Horst CM, Saag MS, Cloud GA, Hamill RJ, Graybill JR, et al. (1997) Treatment of cryptococcal meningitis associated with the acquired immunodeficiency syndrome. National Institute of Allergy and Infectious Diseases Mycoses Study Group and AIDS Clinical Trials Group. N Engl J Med 337: 15-21.

19. Park BJ, Wannemuehler KA, Marston BJ, Govender N, Pappas PG, et al. (2009) Estimation of the current global burden of cryptococcal meningitis among persons living with HIV/AIDS. AIDS 23: 525-530.

20. MacDougall L, Fyfe M, Romney M, Starr M, Galanis E (2011) Risk factors for Cryptococcus gattii infection, British Columbia, Canada. Emerg Infect Dis 17: 193199.

21. Galanis E, Hoang L, Kibsey P, Morshed M, Phillips P (2009) Clinical presentation, diagnosis and management of Cryptococcus gattii cases: Lessons learned from British Columbia. Can J Infect Dis Med Microbiol 20: 23-28.

22. Chen J, Varma A, Diaz MR, Litvintseva AP, Wollenberg KK, et al. (2008) Cryptococcus neoformans strains and infection in apparently immunocompetent patients, China. Emerg Infect Dis 14: 755-762.

23. Ecevit IZ, Glancy CJ, Schmalfuss IM, Nguyen MH (2006) The poor prognosis of central nervous system cryptococcosis among nonimmunosuppressed patients: a call for better disease recognition and evaluation of adjuncts to antifungal therapy. Clin Infect Dis 42: 1443-1447.

24. Lui G, Lee N, Ip M, Choi KW, Tso YK, et al. (2006) Cryptococcosis in apparently immunocompetent patients. QJM 99: 143-151.

25. Nguyen MH, Husain S, Clancy CJ, Peacock JE, Hung CC, et al. (2010) Outcomes of central nervous system cryptococcosis vary with host immune function: results from a multi-center, prospective study. J Infect 61: 419-426.

26. Pappas PG, Perfect JR, Cloud GA, Larsen RA, Pankey GA, et al. (2001) Cryptococcosis in human immunodeficiency virus-negative patients in the era of effective azole therapy. Clin Infect Dis 33: 690-699.

27. Rozenbaum R, Goncalves AJ (1994) Clinical epidemiological study of 171 cases of cryptococcosis. Clin Infect Dis 18: 369-380.

28. Bennett JE, Dismukes WE, Duma RJ, Medoff G, Sande MA, et al. (1979) A comparison of amphotericin $\mathrm{B}$ alone and combined with flucytosine in the treatment of cryptoccal meningitis. N Engl J Med 301: 126-131.

29. Dismukes WE, Cloud G, Gallis HA, Kerkering TM, Medoff G, et al. (1987) Treatment of cryptococcal meningitis with combination amphotericin $\mathrm{B}$ and flucytosine for four as compared with six weeks. N Engl J Med 317: 334-341.

30. Dromer F, Mathoulin S, Dupont B, Brugiere O, Letenneur L (1996) Comparison of the efficacy of amphotericin B and fluconazole in the treatment of cryptococcosis in human immunodeficiency virus-negative patients: retrospective analysis of 83 cases. French Cryptococcosis Study Group. Clin Infect Dis 22 Suppl 2: S154-160.

31. Byrnes EJ, 3rd, Li W, Lewit Y, Perfect JR, Carter DA, et al. (2009) First reported case of Cryptococcus gattii in the Southeastern USA: implications for travelassociated acquisition of an emerging pathogen. PLoS One 4: e5851.

32. Lin X, Heitman J (2006) The biology of the Cryptococcus neoformans species complex. Annu Rev Microbiol 60: 69-105.

33. Singh N, Perfect JR (2007) Immune reconstitution syndrome associated with opportunistic mycoses. Lancet Infect Dis 7: 395-401.

34. Singh N, Alexander BD, Lortholary O, Dromer F, Gupta KL, et al. (2007) Cryptococcus neoformans in organ transplant recipients: impact of calcineurininhibitor agents on mortality. J Infect Dis 195: 756-764.

35. Marchetti O, Moreillon P, Glauser MP, Bille J, Sanglard D (2000) Potent synergism of the combination of fluconazole and cyclosporine in Candida albicans. Antimicrob Agents Chemother 44: 2373-2381.
36. Kontoyiannis DP, Lewis RE, Osherov N, Albert ND, May GS (2003) Combination of caspofungin with inhibitors of the calcineurin pathway attenuates growth in vitro in Aspergillus species. J Antimicrob Chemother 51: 313-316.

37. Kontoyiannis DP, Lewis RE, Alexander BD, Lortholary O, Dromer F, et al. (2008) Calcineurin inhibitor agents interact synergistically with antifungal agents in vitro against Cryptococcus neoformans isolates: correlation with outcome in solid organ transplant recipients with cryptococcosis. Antimicrob Agents Chemother 52: 735-738.

38. Del Poeta M, Cruz MC, Cardenas ME, Perfect JR, Heitman J (2000) Synergistic antifungal activities of bafilomycin $\mathrm{A}(1)$, fluconazole, and the pneumocandin MK-0991/caspofungin acetate (L-743,873) with calcineurin inhibitors FK506 and L-685,818 against Cryptococcus neoformans. Antimicrob Agents Chemother 44: 739-746.

39. Person AK, Kontoyiannis DP, Alexander BD (2010) Fungal infections in transplant and oncology patients. Infect Dis Clin North Am 24: 439-459.

40. Lawn SD, Torok ME, Wood R (2011) Optimum time to start antiretroviral therapy during HIV-associated opportunistic infections. Curr Opin Infect Dis 24: $34-42$

41. Dromer F, Mathoulin-Pelissier S, Launay O, Lortholary O (2007) Determinants of disease presentation and outcome during cryptococcosis: the CryptoA/D study. PLoS Med 4: e21.

42. Lortholary O, Poizat G, Zeller V, Neuville S, Boibieux A, et al. (2006) Longterm outcome of AIDS-associated cryptococcosis in the era of combination antiretroviral therapy. AIDS 20: 2183-2191.

43. Singh N, Dromer F, Perfect JR, Lortholary O (2008) Cryptococcosis in solid organ transplant recipients: current state of the science. Clin Infect Dis 47: 1321-1327.

44. Pappas PG, Alexander BD, Andes DR, Hadley S, Kauffman CA, et al. (2010) Invasive fungal infections among organ transplant recipients: results of the Transplant-Associated Infection Surveillance Network (TRANSNET). Clin Infect Dis 50: 1101-1111.

45. Singh N, Lortholary O, Dromer F, Alexander BD, Gupta KL, et al. (2008) Central nervous system cryptococcosis in solid organ transplant recipients: clinical relevance of abnormal neuroimaging findings. Transplantation 86: 647651.

46. Sun HY, Alexander BD, Lortholary O, Dromer F, Forrest GN, et al. (2009) Lipid formulations of amphotericin B significantly improve outcome in solid organ transplant recipients with central nervous system cryptococcosis. Clin Infect Dis 49: 1721-1728.

47. Wu G, Vilchez RA, Eidelman B, Fung J, Kormos R, et al. (2002) Cryptococcal meningitis: an analysis among 5,521 consecutive organ transplant recipients. Transpl Infect Dis 4: 183-188.

48. Lawn SD, Myer L, Orrell C, Bekker LG, Wood R (2005) Early mortality among adults accessing a community-based antiretroviral service in South Africa: implications for programme design. AIDS 19: 2141-2148.

49. Bicanic T, Muzoora C, Brouwer AE, Meintjes G, Longley N, et al. (2009) Independent association between rate of clearance of infection and clinical outcome of HIV-associated cryptococcal meningitis: analysis of a combined cohort of 262 patients. Clin Infect Dis 49: 702-709.

50. Loyse A, Wilson D, Meintjes G, Jarvis JN, Bicanic T, et al. (2012) Comparison of the early fungicidal activity of high-dose fluconazole, voriconazole, and flucytosine as second-line drugs given in combination with amphotericin B for the treatment of HIV-associated cryptococcal meningitis. Clin Infect Dis 54: $121-128$.

51. Bicanic T, Meintjes G, Rebe K, Williams A, Loyse A, et al. (2009) Immune reconstitution inflammatory syndrome in HIV-associated cryptococcal meningitis: a prospective study. J Acquir Immune Defic Syndr 51: 130-134.

52. Lortholary O, Fontanet A, Memain N, Martin A, Sitbon K, et al, (2005) Incidence and risk factors of immune reconstitution inflammatory syndrome complicating HIV-associated cryptococcosis in France. AIDS 19: 1043-1049.

53. Shelburne SA, Visnegarwala F, Darcourt J, Graviss EA, Giordano TP, et al. (2005) Incidence and risk factors for immune reconstitution inflammatory syndrome during highly active antiretroviral therapy. AIDS 19: 399-406.

54. Sungkanuparph S, Jongwutiwes U, Kiertiburanakul S (2007) Timing of cryptococcal immune reconstitution inflammatory syndrome after antiretroviral therapy in patients with AIDS and cryptococcal meningitis. J Acquir Immune Defic Syndr 45: 595-596.

55. Hamill RJ, Sobel JD, El-Sadr W, Johnson PC, Graybill JR, et al, (2010) Comparison of 2 doses of liposomal amphotericin B and conventional amphotericin B deoxycholate for treatment of AIDS-associated acute cryptococcal meningitis: a randomized, double-blind clinical trial of efficacy and safety. Clin Infect Dis 51: 225-232.

56. Leenders AC, Reiss P, Portegies P, Clezy K, Hop WC, et al. (1997) Liposomal amphotericin B (AmBisome) compared with amphotericin B both followed by oral fluconazole in the treatment of AIDS-associated cryptococcal meningitis. AIDS 11: 1463-1471. 